\title{
Prognostic Nomograms for Nonelderly Adults with Gastric Signet Ring Cell Carcinoma
}

\author{
Hui Wang $\mathbb{D}^{1,2}$ Yao Peng $\mathbb{D}^{1,2}$ Qi Huang $\mathbb{D}^{2}$, Jingjing $W u^{2}$ and Mingjun Zhang $\mathbb{D}^{1,2}$ \\ ${ }^{1}$ Anhui Medical University, Hefei, Anhui, China \\ ${ }^{2}$ Department of Oncology, The Second Affiliated Hospital of Anhui Medical University, Hefei, Anhui, China
}

Correspondence should be addressed to Mingjun Zhang; zhangmjayd@126.com

Received 17 August 2020; Revised 21 January 2021; Accepted 28 February 2021; Published 25 March 2021

Academic Editor: Kyoung-Ho Pyo

Copyright (c) 2021 Hui Wang et al. This is an open access article distributed under the Creative Commons Attribution License, which permits unrestricted use, distribution, and reproduction in any medium, provided the original work is properly cited.

\begin{abstract}
Background. Nomograms were established to predict the survival for gastric signet ring cell carcinoma (GSRC) in young and middle-aged adults. Material and Methods. Eligible patients with GSRC from 2004 to 2015 were collected from the Surveillance, Epidemiology, and End Results (SEER) database and then divided into a training and a testing cohort in proportion. Independent prognostic factors were picked by univariate and multivariate Cox regression analysis to set up nomograms. The predictive effect and clinical value of nomograms were evaluated by the concordance index (C-index), calibration curves, and receiver operating characteristic curve (ROC). Results. A total of 1686 GSRC patients were subsumed into this case for analysis, including a training $(n=1180)$ and a testing cohort $(n=506)$. Independent risk factors related to overall survival (OS) and cancer-specific survival (CSS) comprised of race, TNM stage, tumor size, number of positive lymph nodes (PLNE), and chemotherapy. For OS, the C-indexes of the training and testing cohorts were 0.737 and 0.752 , while for CSS, C-indexes were, respectively, 0.749 and 0.751 . These revealed that nomograms accurately predicted OS and CSS. Calibration curves and ROC demonstrated the apparent superiority of nomograms. Conclusion. We built a well-understood and comprehensive prognostic assessment model for GSRC, which provided an individualized survival prediction in the form of a quantitative score that can be considered for clinical practice.
\end{abstract}

\section{Introduction}

Gastric carcinoma (GA) is part of the most common malignant tumors of the alimentary canal with high morbidity and mortality. According to the statistics, there were approximately 1.033 million new cases and 783,000 deaths worldwide in 2018, seriously endangering human health [1]. Gastric signet ring cell carcinoma (GSRC) is a unique histopathological type of GA, which is rich in distinctive intracytoplasmic mucin components and compression of the surrounding nucleus [2]. The incidence of GSRC increased about tenfold in the United States from 1970 to 2000 (1973: $0.1 / 100,000 ; 2000: 1.4 / 100,000)$, with an average annual increase of 6.5 percent [3].

Relative to non-GSRC, GSRC is characterized by substantial invasion, rapid progression, extreme risk of metasta- sis, and worse prognosis [4]. Young and middle-aged (nonelderly adults) patients are the predominant group of GSRC [5]. The younger GC patients manifest more aggressive behavioral features than older, just like lower curability rate, more inadequate histologic differentiation, and challenging to make a correct diagnosis in the early stage [6-9]. At present, nearly none of the research has considered on the survival of GSRS in nonelderly adults. Therefore, it is necessary to analyze the prognosis of this population, to facilitate clinical individualized treatment and management.

Although the American Joint Committee on Cancer(AJCC-) TNM staging system is the primary basis for evaluating the prognosis and remedy decision of cancer patients, it still has some shortcomings. For example, the effects of additional tumor factors have not been taken into account [10]. Nomogram, as a practical application tool, makes an 
TABLe 1: Patient demographics and clinical characteristics of gastric signet ring cell carcinoma (GSRC) in training and testing cohort.

\begin{tabular}{|c|c|c|c|}
\hline Variables & All patients & Training cohort & Testing cohort \\
\hline Number of patients, $n(\%)$ & $1686(100)$ & $1180(70)$ & $506(30)$ \\
\hline \multicolumn{4}{|l|}{ Age at diagnose (years) } \\
\hline $18-44$ & $401(23.8)$ & $273(23.1)$ & $128(25.3)$ \\
\hline $45-64$ & $1285(76.2)$ & $907(76.9)$ & $378(74.7)$ \\
\hline \multicolumn{4}{|l|}{ Race } \\
\hline Black & $231(13.7)$ & $156(13.2)$ & $75(14.8)$ \\
\hline Other & $362(21.5)$ & $253(21.5)$ & $109(21.6)$ \\
\hline White & $1093(64.8)$ & $771(65.3)$ & $322(63.6)$ \\
\hline \multicolumn{4}{|l|}{ Sex } \\
\hline Female & $807(47.9)$ & $573(48.6)$ & $234(46.2)$ \\
\hline Male & $879(52.1)$ & $607(51.4)$ & $272(53.8)$ \\
\hline \multicolumn{4}{|l|}{ Marital status } \\
\hline Married & $1119(66.4)$ & $785(66.5)$ & $334(66.0)$ \\
\hline Unmarried & $567(33.6)$ & $395(33.5)$ & $172(34.0)$ \\
\hline \multicolumn{4}{|l|}{ Primary site } \\
\hline Proximal third (cardia and fundus) & $290(17.2)$ & $206(17.4)$ & $84(16.6)$ \\
\hline Mid third (body and lesser curvature) & $422(25.0)$ & $303(25.7)$ & $119(23.5)$ \\
\hline Distal third (antrum and pylorus) & $561(33.3)$ & $383(32.5)$ & $178(35.2)$ \\
\hline Greater curvature & $101(6.0)$ & $73(6.2)$ & $28(5.5)$ \\
\hline Overlapping lesions & $184(10.9)$ & $130(11.0)$ & $54(10.7)$ \\
\hline NOS & $128(7.6)$ & $85(7.2)$ & $43(8.5)$ \\
\hline \multicolumn{4}{|l|}{ Grade } \\
\hline Moderately differentiated (II) & $37(2.2)$ & $25(2.1)$ & $12(2.4)$ \\
\hline Poorly differentiated (III) & $1579(93.7)$ & $1104(93.6)$ & $475(93.9)$ \\
\hline Undifferentiated (IV) & $70(4.1)$ & $51(4.3)$ & $19(3.7)$ \\
\hline \multicolumn{4}{|l|}{ T stage } \\
\hline T1 & $276(16.4)$ & $182(15.5)$ & $94(18.6)$ \\
\hline $\mathrm{T} 2$ & $163(9.7)$ & $109(9.2)$ & $54(10.7)$ \\
\hline $\mathrm{T} 3$ & $582(34.5)$ & $426(36.1)$ & $156(30.8)$ \\
\hline $\mathrm{T} 4$ & $665(39.4)$ & $463(39.2)$ & $202(39.9)$ \\
\hline \multicolumn{4}{|l|}{$\mathrm{N}$ stage } \\
\hline No & $486(28.8)$ & $322(27.3)$ & $164(32.4)$ \\
\hline N1 & $298(17.7)$ & $213(18.1)$ & $85(16.8)$ \\
\hline $\mathrm{N} 2$ & $341(20.2)$ & $248(21.0)$ & $93(18.4)$ \\
\hline N3 & $561(33.3)$ & $397(33.6)$ & $164(32.4)$ \\
\hline \multicolumn{4}{|l|}{ M stage } \\
\hline M0 & $1436(85.2)$ & $1005(85.2)$ & $431(85.2)$ \\
\hline M1 & $250(14.8)$ & $175(14.8)$ & $75(14.8)$ \\
\hline \multicolumn{4}{|l|}{ Tumor size $(\mathrm{cm})$} \\
\hline$\leq 5$ & $935(55.5)$ & $651(55.2)$ & $284(56.1)$ \\
\hline$>5$ & $751(44.5)$ & $529(44.8)$ & $222(43.9)$ \\
\hline \multicolumn{4}{|l|}{ PLNE } \\
\hline$<15$ & $1439(85.3)$ & $1001(84.8)$ & $438(86.6)$ \\
\hline$\geq 15$ & $247(14.7)$ & $179(15.2)$ & $68(13.4)$ \\
\hline \multicolumn{4}{|l|}{ Radiation } \\
\hline Yes & $725(43.0)$ & $513(43.5)$ & $212(41.9)$ \\
\hline No/unknown & $961(57.0)$ & $667(56.5)$ & $294(58.1)$ \\
\hline \multicolumn{4}{|l|}{ Chemotherapy } \\
\hline Yes & $1189(70.5)$ & 837 (70.9) & $352(69.6)$ \\
\hline No/unknown & $497(29.5)$ & $343(29.1)$ & $154(30.4)$ \\
\hline
\end{tabular}




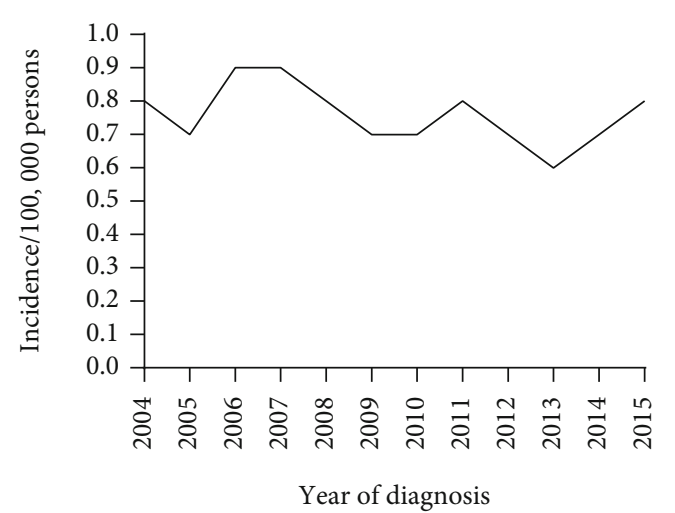

Figure 1: Annual age-adjusted incidence of gastric signet ring cell carcinoma from 2004 to 2015 in 15-64 years old.

individual prediction by quantifying risk factors into scores and shows more accurate and useful than the AJCC-TNM staging system in a variety of cancers [11-13].

Surveillance, Epidemiology, and End Results (SEER) database gathers data on morbidity, diagnosis, treatment, and survival in nearly 27.8 percent of the U.S. population. It is a relatively authoritative platform for studying the general peculiarity of carcinoma [14]. Based on the SEER, this study explored the factors influencing prognosis in 18-64 years old patients with GSRC, then attempted to develop nomograms for predicting cancer-specific survival (CSS) and overall survival (OS) of such patients, hoping to assist medical professionals to make more personalized and correct judgment in clinical practice.

\section{Patients and Methods}

2.1. Patients. We selected suitable patients from the SEER database (1975-2016 varying) using the SEER $*$ Stat software (version 8.3.6, http://seer.cancer.gov/seerstat/).

The specific inclusion criteria were (1) the period of diagnosis was from 2004 to 2015; (2) the histological pathologic confirmation was limited to adenocarcinoma, which was further stratified as signet ring cell carcinoma (SRC) (using ICD-O-3 histology code, 8490/3) or non-SRC of the adenocarcinoma (8140/3); (3) the primary site was limited to stomach (C16.0-16.9); and (4) the age $\geq 18$ years old and $<65$ years old.

The exclusion criteria were (1) more than one primary tumor or combined with other tumors, (2) patient survived less than one month or was unknown, and (3) the necessary information covered (as follows) was lacking.

2.2. Clinical Variables. For each case, tumor stage was redescribed according to the 7th AJCC-TNM staging system.

The following clinicopathological variables were extracted: race, sex, pathologic grade, marital status, age at diagnosis, year of diagnosis, primary site, TNM stage, tumor size, number of positive lymph nodes (PLNE), radiotherapy (RT), chemotherapy, cause-specific death classification, survival months, survival status, and so on.
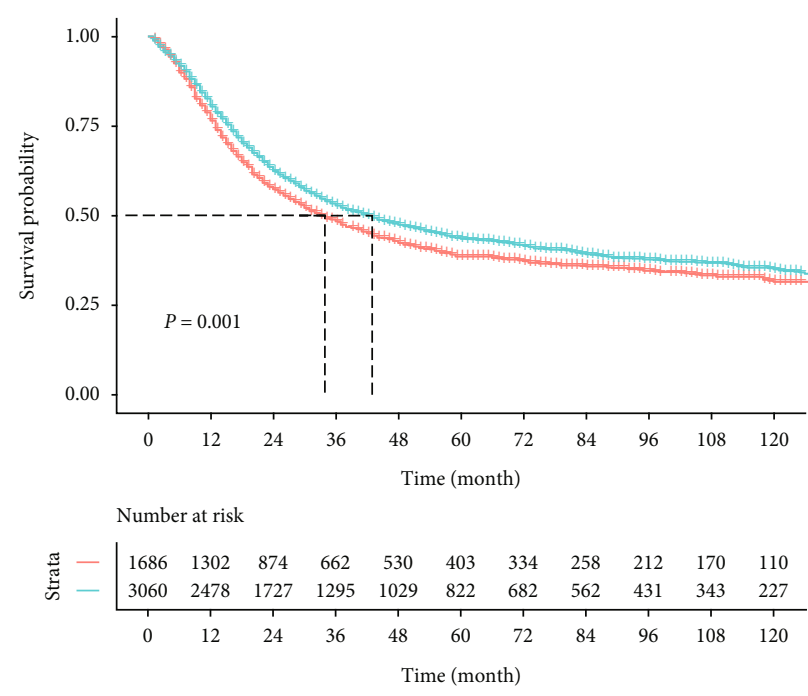

Strata

+ Group $=$ GSRCA

+ Group $=$ non-GSRCA

(a)

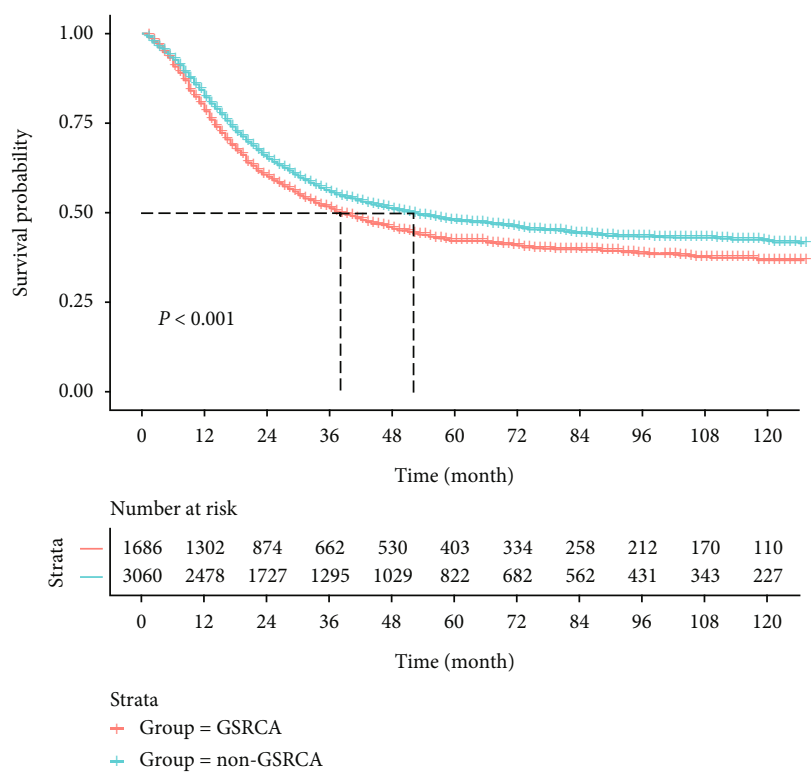

(b)

FIGURE 2: Survival analysis of GSRC and non-GSRC: (a) OS and (b) CSS were shown for all patients.

Race categories were split into black, other (including Chinese, Japanese, Korean, and Native Hawaiian/Pacific Islander and those who reported multiple categories), and white. The primary site was further subdivided into the proximal third (C16.0 cardia and C16.1 fundus), the mid third (C16.2 body and C16.5 lesser curvature), the distal third (C16.3 antrum and C16.4 pylorus), greater curvature (C16.6), overlapping lesions of the stomach (C16.8), and NOS (C16.9).

2.3. Survival Analysis. The survival curves of OS and CSS were drawn by Kaplan-Meier function, and log-rank was used to test the difference in survival rate among different groups. 

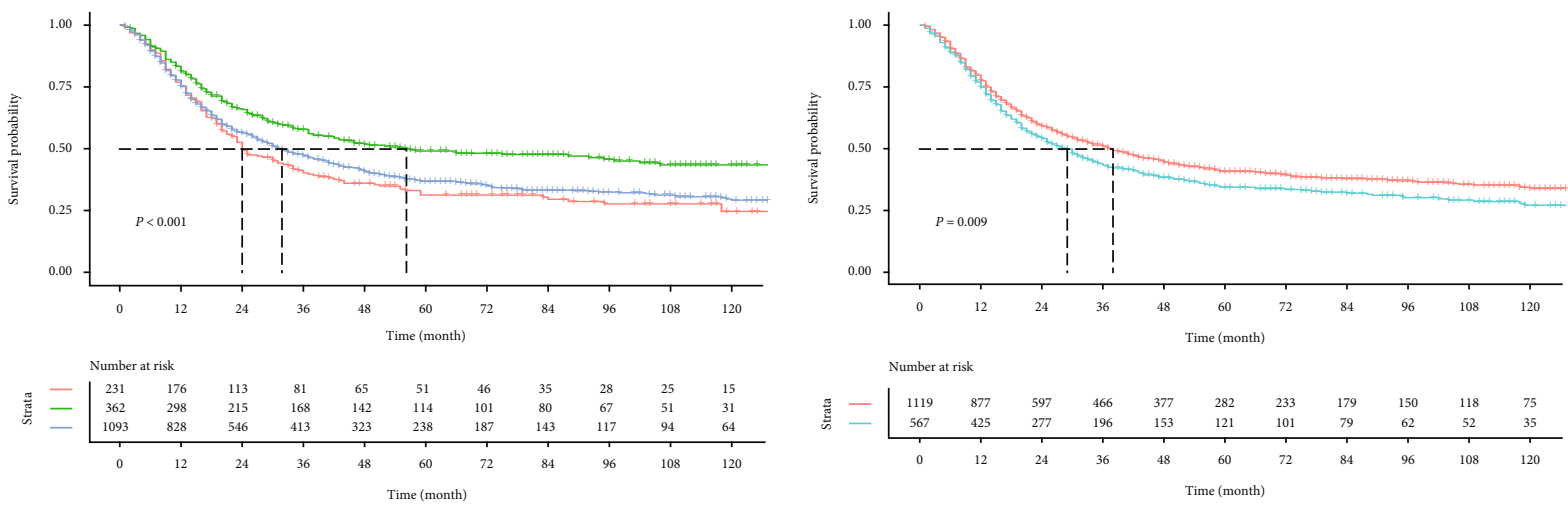

Strata

+ Race $=$ black

+ Race $=$ other

$-$

us $=$ married

(a)

(b)
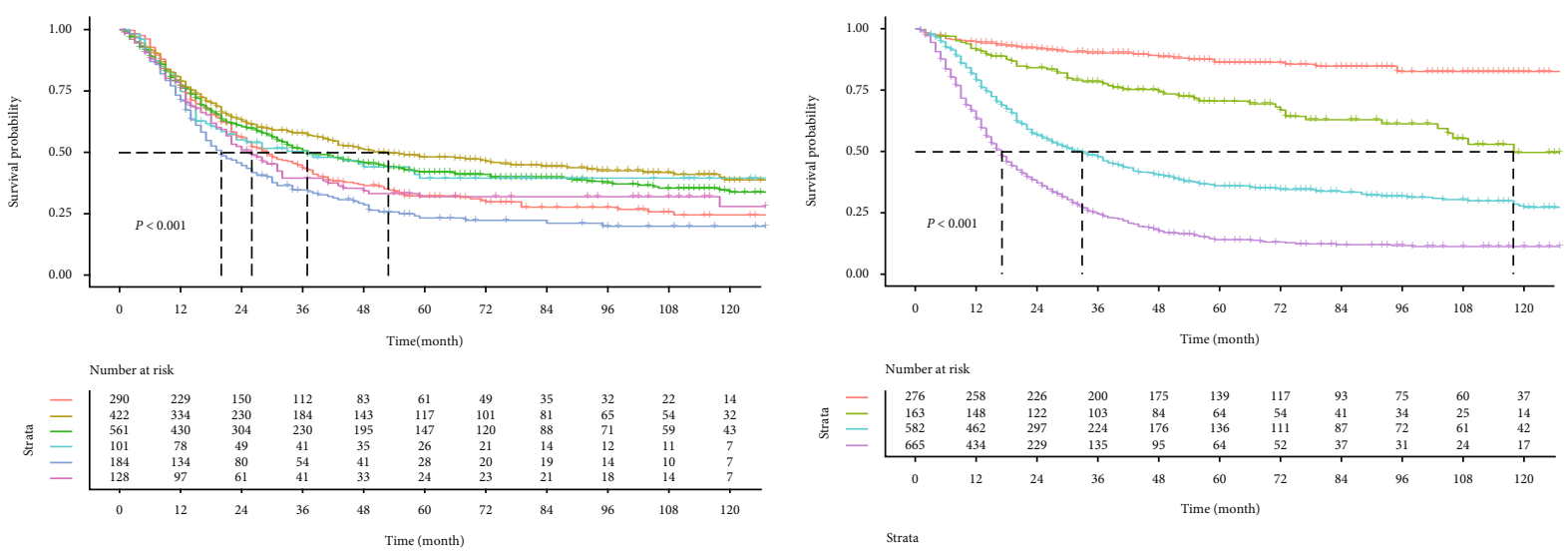

Strata

- Primary site $=$ greater curvature

- Primary site $=$ proximal thi

+ Primary site $=$ distal third

Primary site $=$ NOS

$+\quad \mathrm{T}$ stage $=\mathrm{T} 1$

$+\mathrm{T}$ stage $=\mathrm{T} 3$

$+\mathrm{T}$ stage $=\mathrm{T} 2$

$+\mathrm{T}$ stage $=\mathrm{T} 4$

(c)

(d)
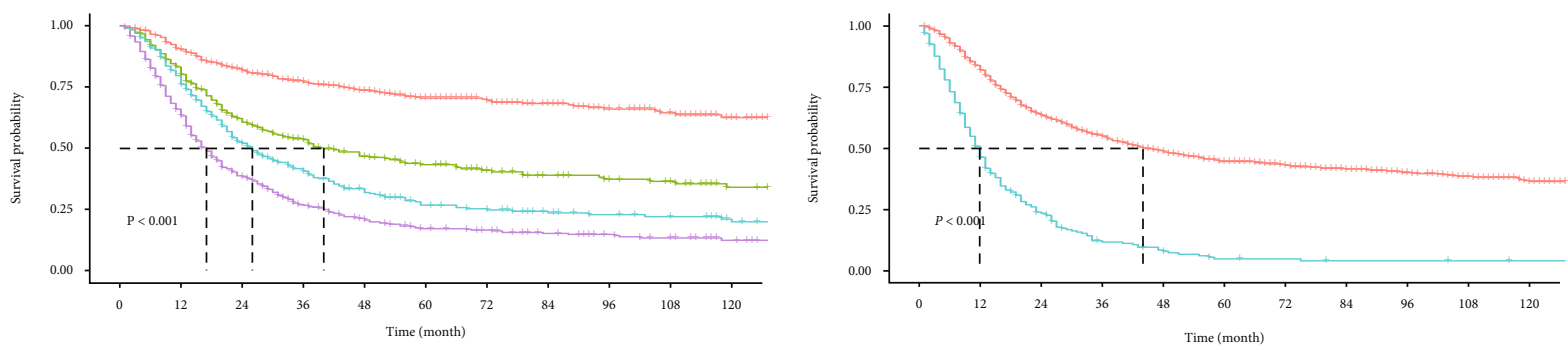

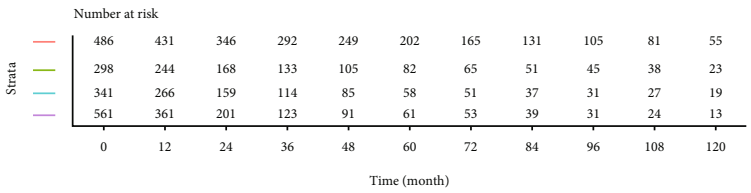

Strata

$\begin{array}{ll}\text { Strata } & \\ +\mathrm{N} \text { stage }=\mathrm{N} 0 & +\mathrm{N} \text { stage }=\mathrm{N} 2 \\ +\mathrm{N} \text { stage }=\mathrm{N} 1 & +\mathrm{N} \text { stage }=\mathrm{N} 3\end{array}$

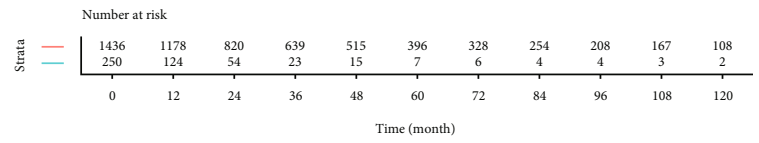

Strata

$+\mathrm{M}$ stage $=\mathrm{M} 0$

(e)

Figure 3: Continued. 


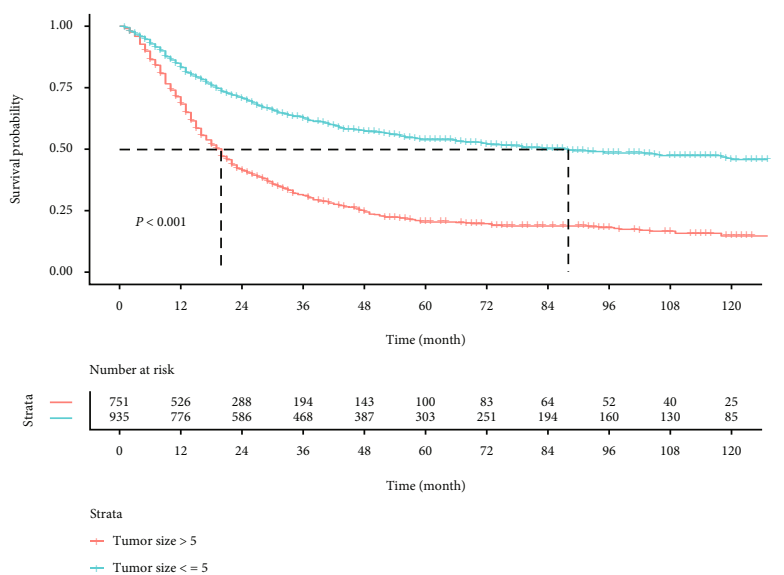

(g)
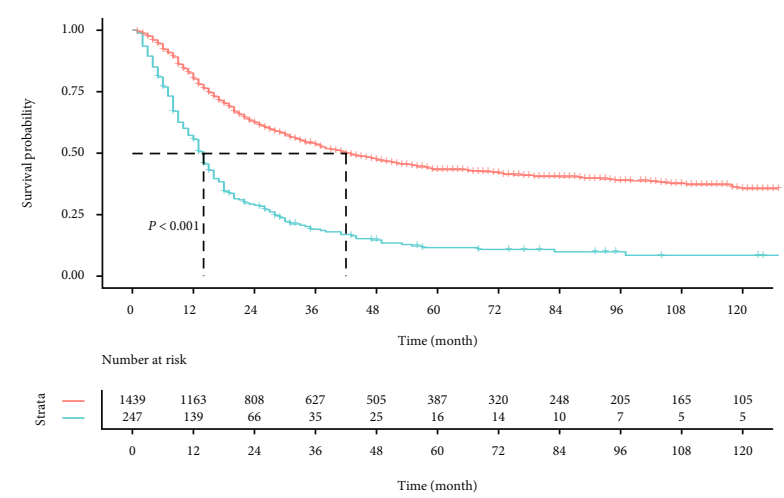

Strata

+ PLNE $<15$

+ PLNE $>=15$

(h)

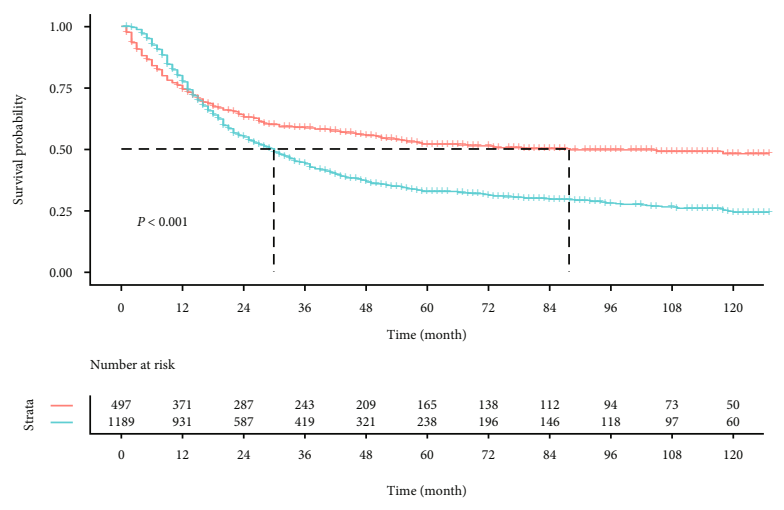

Strata
$+\quad$ Chemotherapy $=$ no/ unknown

$+\quad$ Chemotherapy $=$ yes

(i)

FIGURE 3: Kaplan-Meier survival analysis of OS for GSRC stratified by (a) race, (b) marital status, (c) primary site, (d) T stage, (e) N stage, (f) M stage, (g) tumor size, (h) PLNE, and (i) chemotherapy.

2.4. Construction of the Nomograms. All applicable patients were randomized into the training and testing cohort in a ratio of $7: 3$. Nomograms were designed by the training cohort. Univariate and multivariate Cox regression analyses were used to screen out and determine significant independent prognostic risk factors for CSS and OS. Then, we incorporated these factors into construction nomograms.

2.5. Validation of the Nomograms. Validation of the nomograms was primarily implemented in both the training and testing cohorts. The Harrell's concordance index (C-index) and calibration curves were applied to evaluate the predictive performance of the nomograms. The higher the Cindex was, the more accurate the prediction. Calibration curves were generated to visually judge the consistency between the predicted and actual probability of survival on the basis of bootstrap 1000 resamples [15-17]. The receiver operating characteristic curve (ROC) and the area under ROC (AUC) were also used to assess the precision and specificity of nomograms.

Survival rates were calculated using SPSS 22.0 Statistical Package, the age-adjusted incidence of GSRC aged 15-64 years from 2004 to 2015 was analyzed by GraphPad Prism 8 , and the rest part of statistical analysis was undertaken using the $\mathrm{R}$ software (version 3.6.1, http://www.r-project . org/). The $P$ value $<0.05$ was deemed statistically significant.

\section{Results}

3.1. Patient Characteristics. 1686 suitable cases with GSRC and 3060 instances with non-GSRC were contained in this process from 2004 to 2015 (Supplementary Table 1). Table 1 presented basic information about GSRC in both training $(n=1180)$ and testing $(n=506)$ cohorts, including demographics, clinical characteristics, and treatment condition. In terms of the entire study cohort, there were 879 males and 807 females. White (64.8\%), married (66.4\%), middle-aged (23.8\%), and poor differentiation (93.7\%) accounted for predominance. With regard to the primary site of tumor, the most familiar location was the distal third gastric region, occupying approximately $33.3 \%$, followed by the mid third gastric region. In addition to chemotherapy, which became even more common therapy for all patients, and many patients had also received RT.

Figure 1 revealed the age-adjusted incidence of GSRC in 15-64 years old from 2004 to 2015, showing a relatively stable rate of $0.6-0.9 / 100,000$ persons. We can more intuitively 

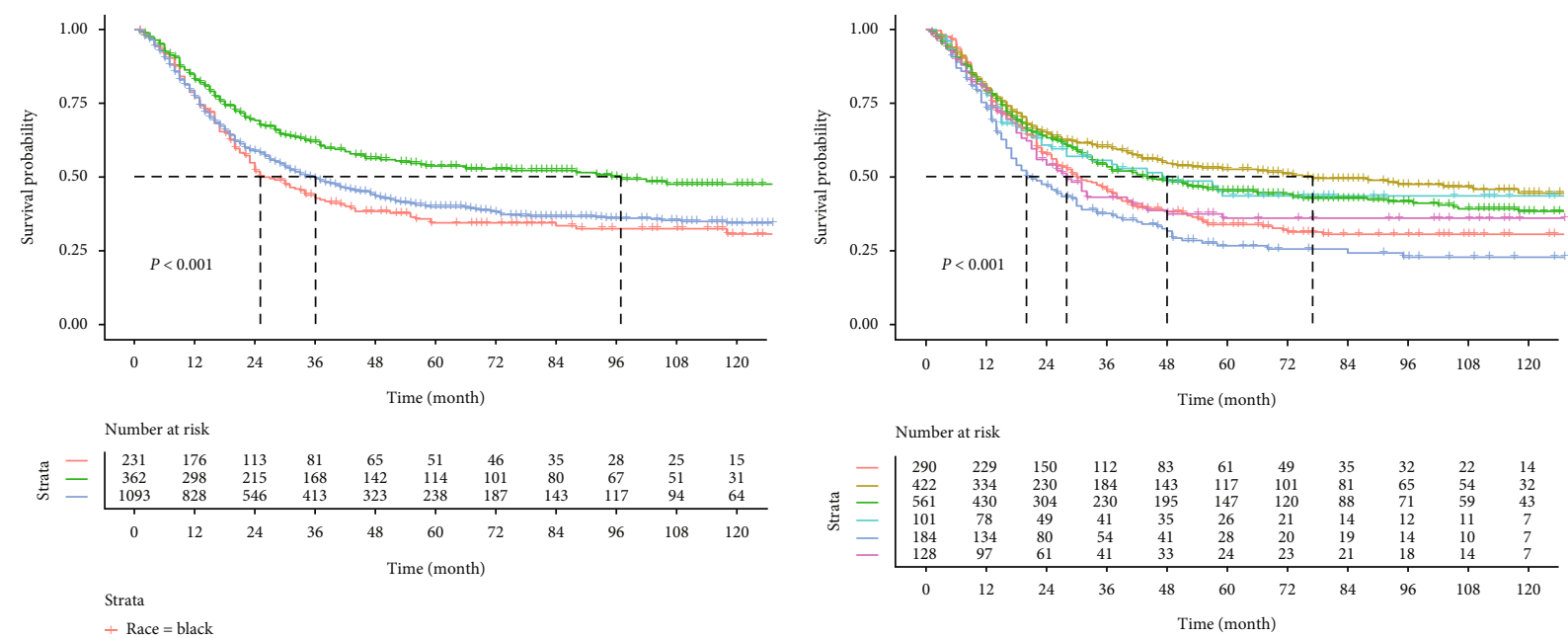

+ Race $=$ black

+ Race $=$ other

Strata

+ Primary site $=$ proximal third

+ Primary site $=$ mid third

+ Primary site $=$ greater curvature

- Primary site $=$ distal thir

- Primary site $=$ overlapping lesions

(a)

(b)
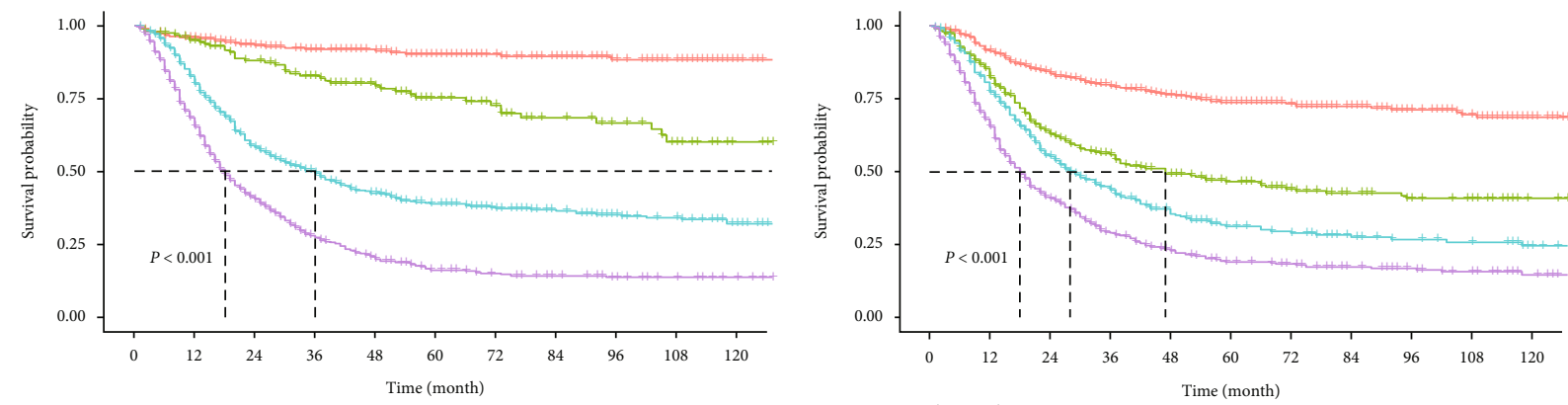

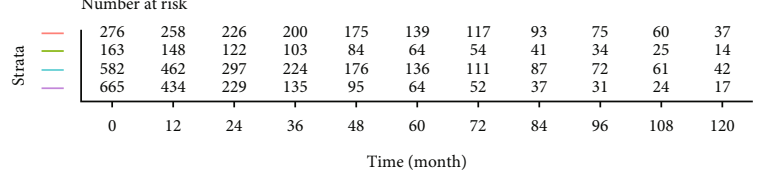

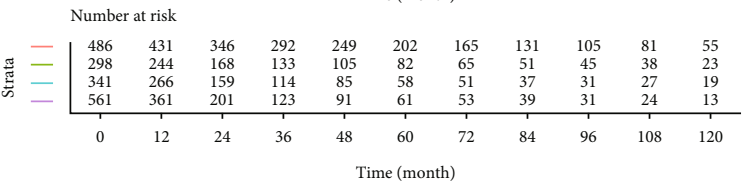

Strata

$+\mathrm{T}$ stage $=\mathrm{T} 1$

$+\mathrm{T}$ stage $=\mathrm{T} 3$

Strata

$+\mathrm{T}$ stage $=\mathrm{T} 2+\mathrm{T}$ stage $=\mathrm{T} 4$

$+\quad \mathrm{N}$ stage $=\mathrm{N} 0$

$+\mathrm{N}$ stage $=\mathrm{N} 1$

$+\quad \mathrm{N}$ stage $=\mathrm{N} 2$

$+\quad \mathrm{N}$ stage $=\mathrm{N} 3$

(c)

(d)

Figure 4: Continued. 

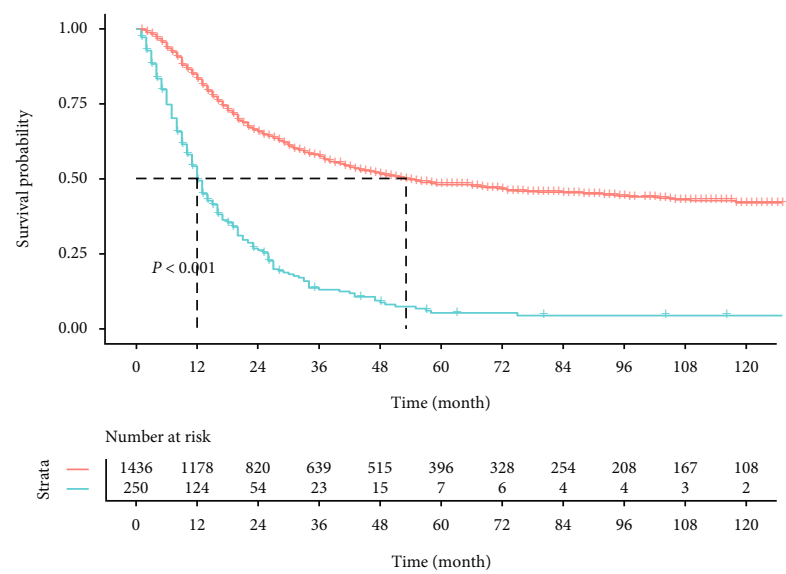

Strata

$+\mathrm{M}$ stage $=\mathrm{M} 0$

$+\mathrm{M}$ stage $=\mathrm{Ml}$

(e)
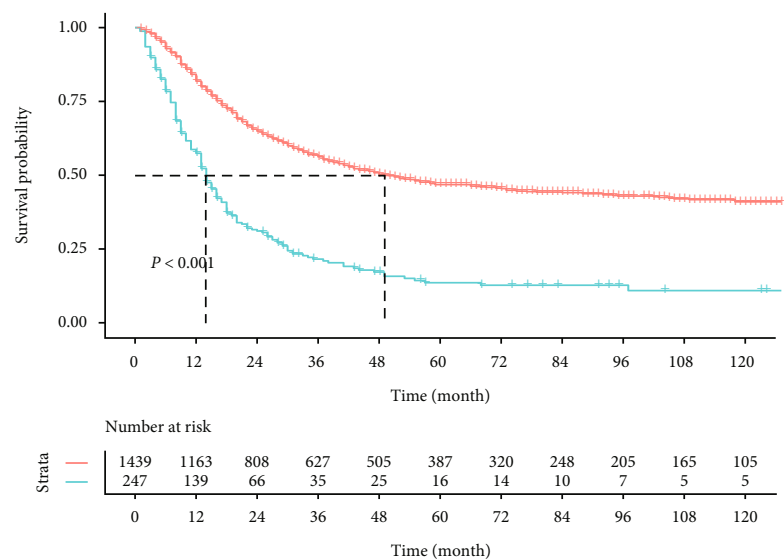

Strata

+ PLNE $<15$

+ PLNE $>=15$

(g)
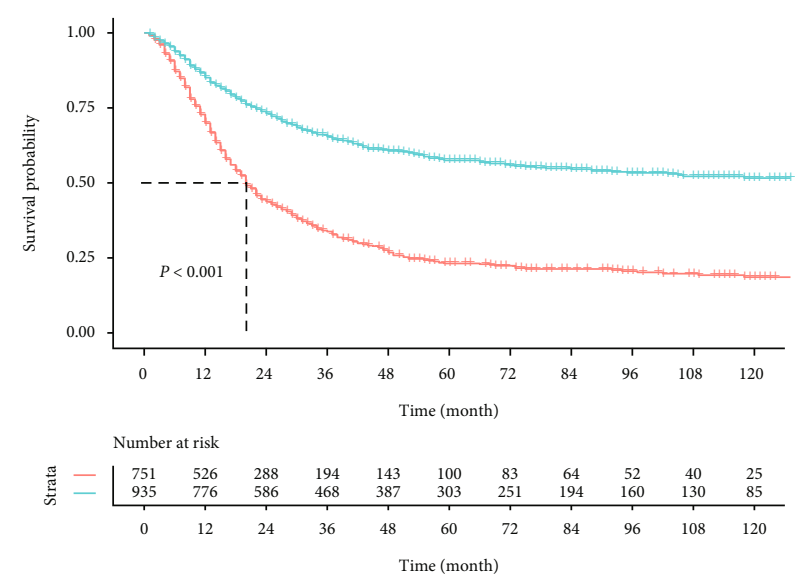

Strata

+ Tumor size $>5$

+ Tumor size $<=5$

(f)

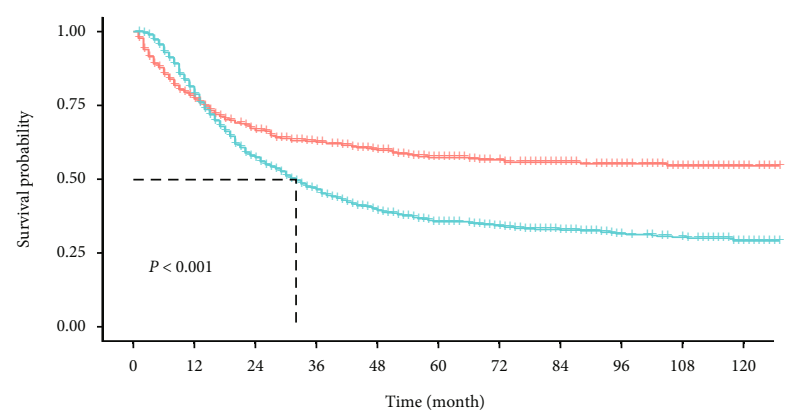

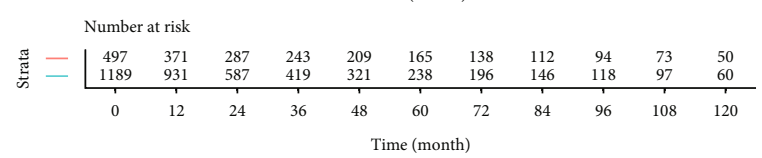

Strata

+ Chemotherapy $=$ no/unknown

+ Chemotherapy $=$ yes

(h)

FIgURE 4: Kaplan-Meier survival analysis of CSS for GSRC stratified by (a) race, (b) primary site, (c) T stage, (d) N stage, (e) M stage, (f) tumor size, (g) PLNE, and (h) chemotherapy.

understand the incidence trend of the disease in nonelderly patients.

3.2. Survival. In the whole cohort, at the end of follow-up, 993 patients died in all. Kaplan-Meier survival analysis displayed that the OS rates of patients with GSRC in 3, 5, and 10 years were $48.5 \%, 38.7 \%$, and $31.6 \%$, and the CSS rates were $51.2 \%$, $42.1 \%$, and $36.7 \%$, respectively. Median OS and CSS were 34 and 38 months. The OS rates of 3, 5, and 10 years were $52.9 \%$, $43.6 \%$, and $35.1 \%$ who were non-GSRC patients, and the CSS rates were $55.7 \%, 47.7 \%$, and $42.1 \%$. GSRC acquired a more unfavourable outcome than non-GSRC in 18-64 years old (Figure 2(a), OS; Figure 2(b), CSS).

Figures 3 and 4 severally discovered the Kaplan-Meier survival curves of OS and CSS for some pathological factors of GSRC, which were meaningful to survival. Patients whose primary site was located in the mid third gastric region, the OS and CSS were longer. When the tumor was progressed, the PLNE was increased, and the prognosis was poorer. Race and chemotherapy were also important factors affecting sur- vival. Married patients inclined to gain better OS than unmarried patients but did not correlate with CSS. Besides, sex, grade, RT, and age at diagnosis were no significant impact on OS or CSS.

\subsection{Construction of Prognostic Nomograms for OS and CSS.} Through the univariate and multivariate Cox regression analysis, finally, we obtained seven independent prognostic risk factors of OS and CSS, including race, TNM stage, tumor size, PLNE, and chemotherapy (Table 2, OS; Table 3, CSS).

In the whole study set, nomograms were established according to all of the independent prognostic factors stemmed from multivariate Cox regression analysis in the training cohort. We were able to intuitively estimate the probabilities of OS (Figure 5(a)) and CSS (Figure 5(b)) for 3,5 , and 10 years by adding the scores related to each variable and predicting the total points to the bottom.

3.4. Validation of Prognostic Nomograms for OS and CSS. The nomograms which were validated showed excellent accuracy. 
TABLE 2: Univariate and multivariate Cox analysis of the determinants of overall survival (OS) of patients with gastric signet ring cell carcinoma (GSRC).

\begin{tabular}{|c|c|c|c|c|}
\hline \multirow{2}{*}{ Variables } & \multicolumn{2}{|c|}{ Univariate analysis } & \multicolumn{2}{|c|}{ Multivariate analysis } \\
\hline & HR $(95 \% \mathrm{CI})$ & $P$ value & HR $(95 \% \mathrm{CI})$ & $P$ value \\
\hline \multicolumn{5}{|l|}{ Age at diagnose (years) } \\
\hline $18-44$ & 1 & & & \\
\hline $45-64$ & $1.118(0.937-1.334)$ & 0.215 & & \\
\hline \multicolumn{5}{|l|}{ Race } \\
\hline Black & 1 & & 1 & \\
\hline Other & $0.672(0.517-0.873)$ & 0.003 & $0.658(0.501-0.864)$ & 0.003 \\
\hline White & $0.948(0.765-1.174)$ & 0.624 & $0.870(0.694-1.090)$ & 0.226 \\
\hline \multicolumn{5}{|l|}{ Sex } \\
\hline Female & 1 & & & \\
\hline Male & $0.974(0.840-1.129)$ & 0.722 & & \\
\hline \multicolumn{5}{|l|}{ Marital status } \\
\hline Married & 1 & & 1 & \\
\hline Unmarried & $1.210(1.036-1.412)$ & 0.016 & $1.108(0.943-1.301)$ & 0.214 \\
\hline \multicolumn{5}{|l|}{ Primary site } \\
\hline Proximal third (cardia and fundus) & 1 & & 1 & \\
\hline Mid third (body and lesser curvature) & $0.741(0.588-0.934)$ & 0.011 & $0.815(0.642-1.035)$ & 0.094 \\
\hline Distal third (antrum and pylorus) & $0.882(0.713-1.092)$ & 0.249 & $0.831(0.664-1.040)$ & 0.106 \\
\hline Greater curvature & $0.932(0.661-1.313)$ & 0.687 & $0.887(0.625-1.258)$ & 0.500 \\
\hline Overlapping lesions & $1.314(1.012-1.705)$ & 0.040 & $0.808(0.613-1.065)$ & 0.130 \\
\hline NOS & $0.985(0.712-1.362)$ & 0.927 & $0.870(0.623-1.215)$ & 0.413 \\
\hline \multicolumn{5}{|l|}{ Grade } \\
\hline Moderately differentiated (II) & 1 & & & \\
\hline Poorly differentiated (III) & $1.230(0.695-2.178)$ & 0.477 & & \\
\hline Undifferentiated (IV) & $1.362(0.701-2.645)$ & 0.362 & & \\
\hline \multicolumn{5}{|l|}{ T stage } \\
\hline $\mathrm{T} 1$ & 1 & & 1 & \\
\hline $\mathrm{T} 2$ & $2.624(1.598-4.310)$ & $<0.001$ & $2.562(1.534-4.278)$ & $<0.001$ \\
\hline $\mathrm{T} 3$ & $5.877(3.951-8.740)$ & $<0.001$ & $4.668(3.028-7.195)$ & $<0.001$ \\
\hline $\mathrm{T} 4$ & $11.257(7.604-16.670)$ & $<0.001$ & $7.353(4.740-11.406)$ & $<0.001$ \\
\hline \multicolumn{5}{|l|}{$\mathrm{N}$ stage } \\
\hline No & 1 & & 1 & \\
\hline N1 & $2.189(1.678-2.854)$ & $<0.001$ & $1.327(1.001-1.759)$ & 0.049 \\
\hline $\mathrm{N} 2$ & $2.689(2.091-3.458)$ & $<0.001$ & $1.528(1.166-2.002)$ & 0.002 \\
\hline N3 & $4.496(3.580-5.647)$ & $<0.001$ & $1.724(1.312-2.265)$ & $<0.001$ \\
\hline \multicolumn{5}{|l|}{ M stage } \\
\hline M0 & 1 & & 1 & \\
\hline M1 & $3.547(2.958-4.254)$ & $<0.001$ & $2.113(1.746-2.557)$ & $<0.001$ \\
\hline \multicolumn{5}{|l|}{ Tumor size $(\mathrm{cm})$} \\
\hline$\leq 5$ & 1 & & 1 & \\
\hline$>5$ & $2.338(2.010-2.720)$ & $<0.001$ & $1.276(1.082-1.504)$ & 0.004 \\
\hline \multicolumn{5}{|l|}{ PLNE } \\
\hline$<15$ & 1 & & 1 & \\
\hline$\geq 15$ & $2.800(2.335-3.357)$ & $<0.001$ & $1.418(1.135-1.771)$ & 0.002 \\
\hline \multicolumn{5}{|l|}{ Radiation } \\
\hline No/unknown & 1 & & & \\
\hline Yes & $0.909(0.784-1.055)$ & 0.211 & & \\
\hline \multicolumn{5}{|l|}{ Chemotherapy } \\
\hline No/unknown & 1 & & 1 & \\
\hline Yes & $1.399(1.176-1.666)$ & $<0.001$ & $0.669(0.555-0.807)$ & $<0.001$ \\
\hline Year of diagnose & $0.964(0.942-0.987)$ & 0.002 & $1.000(0.976-1.025)$ & 0.987 \\
\hline
\end{tabular}

HR: hazard ratio; CI: confidence interval; PLNE: number of positive lymph nodes. 
TABLE 3: Univariate and multivariate Cox analysis of the determinants of cancer-specific survival (CSS) of patients with gastric signet ring cell carcinoma (GSRC).

\begin{tabular}{|c|c|c|c|c|}
\hline \multirow{2}{*}{ Variables } & \multicolumn{2}{|c|}{ Univariate analysis } & \multicolumn{2}{|c|}{ Multivariate analysis } \\
\hline & HR (95\% CI) & $P$ value & HR (95\% CI) & $P$ value \\
\hline \multicolumn{5}{|l|}{ Age at diagnose (years) } \\
\hline $18-44$ & 1 & & & \\
\hline $45-64$ & $1.127(0.935-1.358)$ & 0.209 & & \\
\hline \multicolumn{5}{|l|}{ Race } \\
\hline Black & 1 & & 1 & \\
\hline Other & $0.644(0.487-0.853)$ & 0.002 & $0.613(0.462-0.813)$ & $<0.001$ \\
\hline White & $0.967(0.771-1.212)$ & 0.769 & $0.859(0.681-1.082)$ & 0.197 \\
\hline \multicolumn{5}{|l|}{ Sex } \\
\hline Female & 1 & & & \\
\hline Male & $0.969(0.829-1.132)$ & 0.688 & & \\
\hline \multicolumn{5}{|l|}{ Marital status } \\
\hline Married & 1 & & & \\
\hline Unmarried & $1.170(0.993-1.378)$ & 0.060 & & \\
\hline \multicolumn{5}{|l|}{ Primary site } \\
\hline Proximal third (cardia and fundus) & 1 & & 1 & \\
\hline Mid third (body and lesser curvature) & $0.732(0.573-0.934)$ & 0.012 & $0.811(0.630-1.045)$ & 0.105 \\
\hline Distal third (antrum and pylorus) & $0.880(0.703-1.101)$ & 0.263 & $0.835(0.659-1.057)$ & 0.134 \\
\hline Greater curvature & $0.886(0.613-1.279)$ & 0.518 & $0.840(0.578-1.222)$ & 0.362 \\
\hline Overlapping lesions & $1.347(1.027-1.769)$ & 0.032 & $0.818(0.613-1.092)$ & 0.174 \\
\hline NOS & $0.961(0.681-1.356)$ & 0.822 & $0.853(0.598-1.217)$ & 0.381 \\
\hline \multicolumn{5}{|l|}{ Grade } \\
\hline Moderately differentiated (II) & 1 & & & \\
\hline Poorly differentiated (III) & $1.207(0.665-2.193)$ & 0.536 & & \\
\hline Undifferentiated (IV) & $1.398(0.701-2.791)$ & 0.342 & & \\
\hline \multicolumn{5}{|l|}{ T stage } \\
\hline $\mathrm{T} 1$ & 1 & & 1 & \\
\hline $\mathrm{T} 2$ & $2.957(1.636-5.347)$ & $<0.001$ & $2.760(1.502-5.072)$ & 0.001 \\
\hline $\mathrm{T} 3$ & $7.929(4.907-12.812)$ & $<0.001$ & $5.951(3.557-9.955)$ & $<0.001$ \\
\hline $\mathrm{T} 4$ & $15.347(9.540-24.688)$ & $<0.001$ & $9.408(5.590-15.836)$ & $<0.001$ \\
\hline \multicolumn{5}{|l|}{$\mathrm{N}$ stage } \\
\hline No & 1 & & 1 & \\
\hline N1 & $2.350(1.760-3.138)$ & $<0.001$ & $1.345(0.992-1.823)$ & 0.057 \\
\hline $\mathrm{N} 2$ & $2.926(2.225-3.848)$ & $<0.001$ & $1.555(1.163-2.080)$ & 0.003 \\
\hline N3 & $5.091(3.972-6.525)$ & $<0.001$ & $1.780(1.332-2.379)$ & $<0.001$ \\
\hline \multicolumn{5}{|l|}{ M stage } \\
\hline M0 & 1 & & 1 & \\
\hline M1 & $3.764(3.119-4.542)$ & $<0.001$ & $2.193(1.801-2.670)$ & $<0.001$ \\
\hline \multicolumn{5}{|l|}{ Tumor size $(\mathrm{cm})$} \\
\hline$\leq 5$ & 1 & & 1 & \\
\hline$>5$ & $2.448(2.086-2.873)$ & $<0.001$ & $1.278(1.076-1.519)$ & 0.005 \\
\hline \multicolumn{5}{|l|}{ PLNE } \\
\hline$<15$ & 1 & & 1 & \\
\hline$\geq 15$ & $2.910(2.410-3.515)$ & $<0.001$ & $1.428(1.135-1.797)$ & 0.002 \\
\hline \multicolumn{5}{|l|}{ Radiation } \\
\hline No/unknown & 1 & & & \\
\hline Yes & $0.898(0.768-1.051)$ & 0.179 & & \\
\hline \multicolumn{5}{|l|}{ Chemotherapy } \\
\hline No/unknown & 1 & & 1 & \\
\hline Yes & $1.492(1.237-1.800)$ & $<0.001$ & $0.698(0.572-0.851)$ & $<0.001$ \\
\hline Year of diagnose & $0.958(0.935-0.982)$ & $<0.001$ & $0.996(0.971-1.022)$ & 0.742 \\
\hline
\end{tabular}

HR: hazard ratio; CI: confidence interval; PLNE: number of positive lymph nodes. 


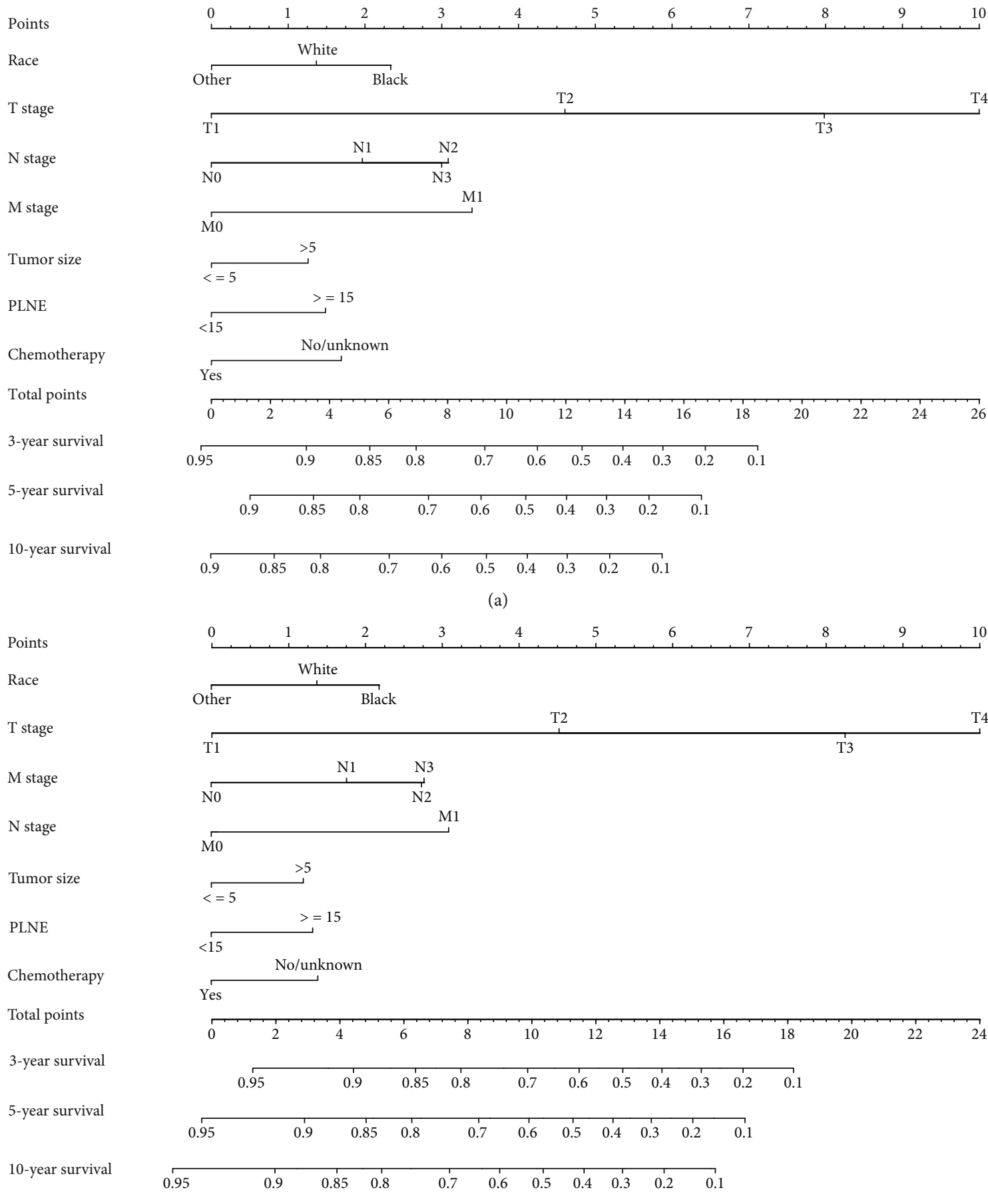

(b)

FIGURE 5: Nomograms for predicting the 3-, 5-, and 10-year OS (a) and CSS (b) of patients with GSRC in 18-64 years old.

When validation of nomograms was performed in the training cohort for OS and CSS, the C-indexes were, respectively, 0.737 and 0.749 . The $\mathrm{C}$-indexes for the nomograms to predict OS and CSS were, respectively, 0.752 and 0.751 in the testing cohort.

Through the calibration curves for probabilities of OS and CSS, predictions by the nomograms were in optimal correlation with actual survival observation in training (Figure 6) and testing cohort (Figure 7). Figure 8 illustrated that the values of AUC indicated a satisfactory ability to pre- dict survival, which was all over 0.8 (0.81-0.85 in the training cohort; $0.85-0.87$ in the testing cohort). To sum up, nomograms that we built showed considerable reliability.

\section{Discussion}

GSRC is a highly malignant type of GA, with a reported fiveyear survival rate of only $15.9 \%$ [18]. The age of high incidence is generally less than 65 years old in GSRC. Nevertheless, survival analysis of this population continues to be 


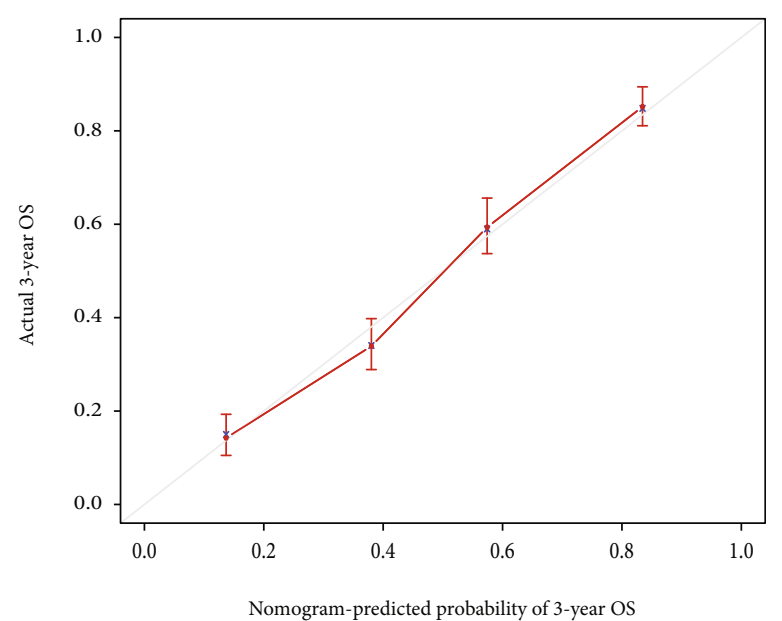

(a)

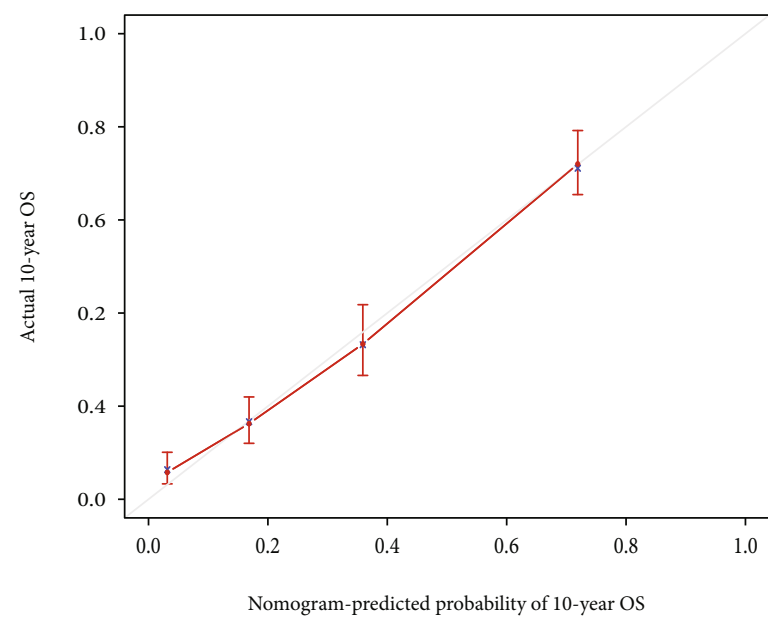

(c)

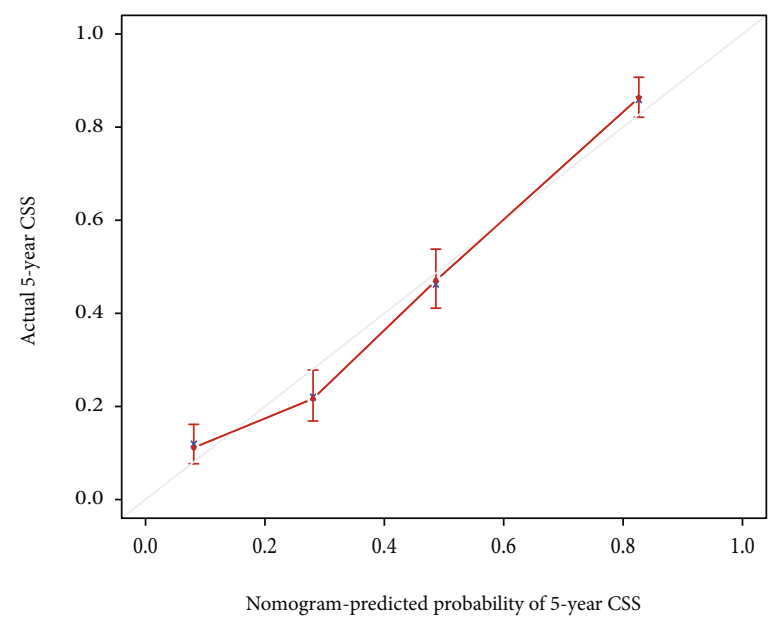

(e)

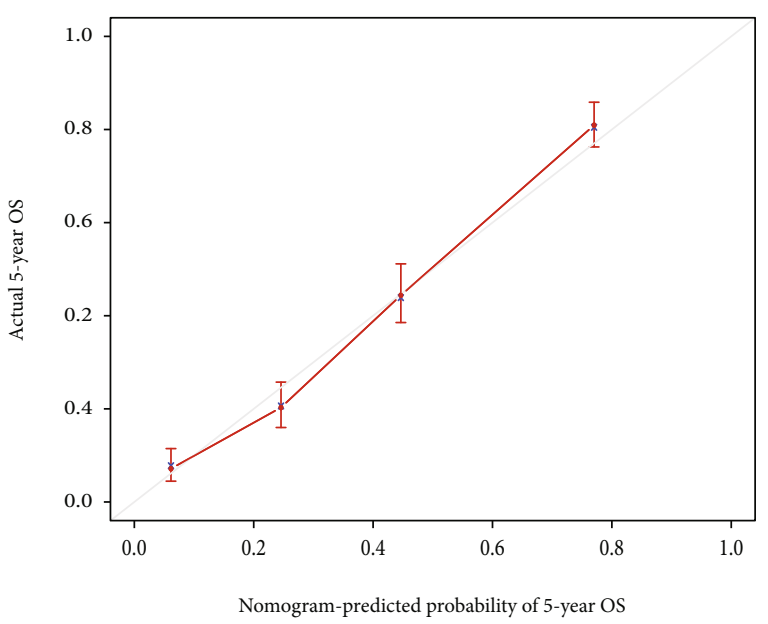

(b)

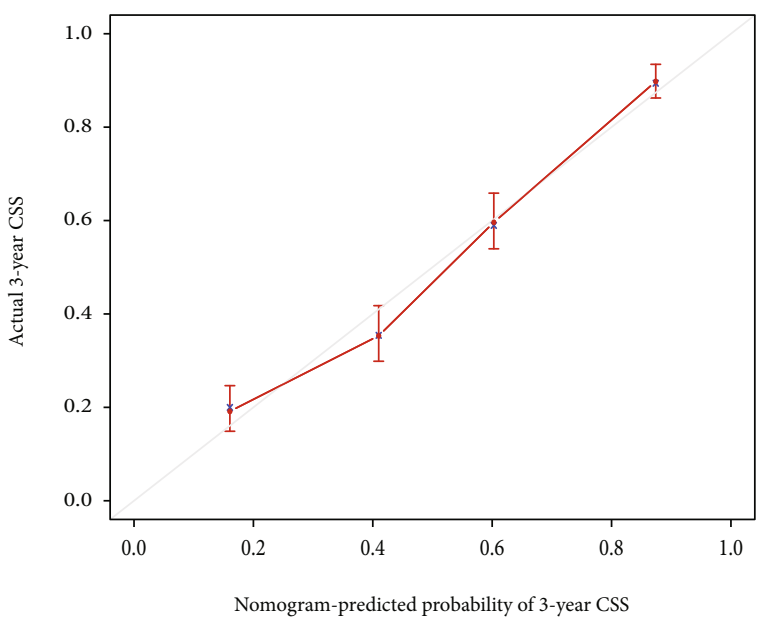

(d)

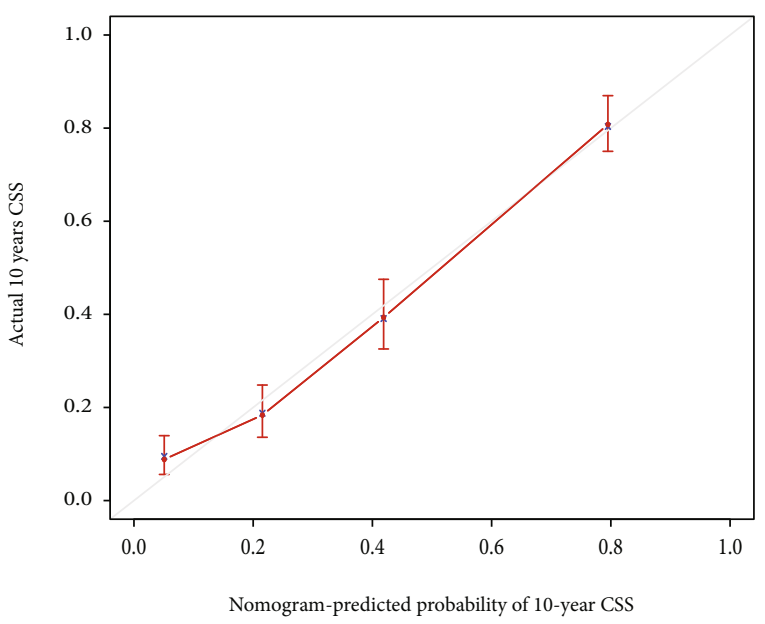

(f)

FIGURE 6: Calibration curves of the nomogram for 3-, 5-, and 10-year OS (a-c) and CSS (d-f) in training cohort.

scarce. Hence, it is urgent to make an in-depth study on the prognostic factors and establish a predictive model to guide the clinical work better. Thus, on the strength of a large population cohort, we described the prognostic factors and constructed nomograms to predict OS and CSS for GSRC patients aged 18-64 years.
For the Asians, Native Americans, and Pacific Islanders, the prognosis was slightly better than blacks and whites. This was in line with the research by Wang et al. [19]. Several studies had come to similar conclusions. Kim et al. studied 13084 patients with gastric adenocarcinoma in the Los Angeles County Cancer Surveillance Program and found that 


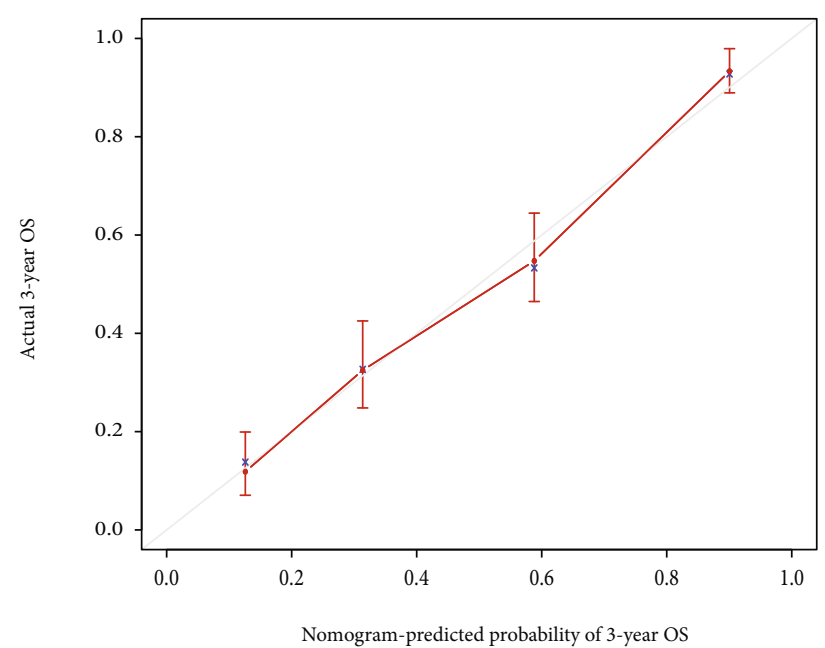

(a)

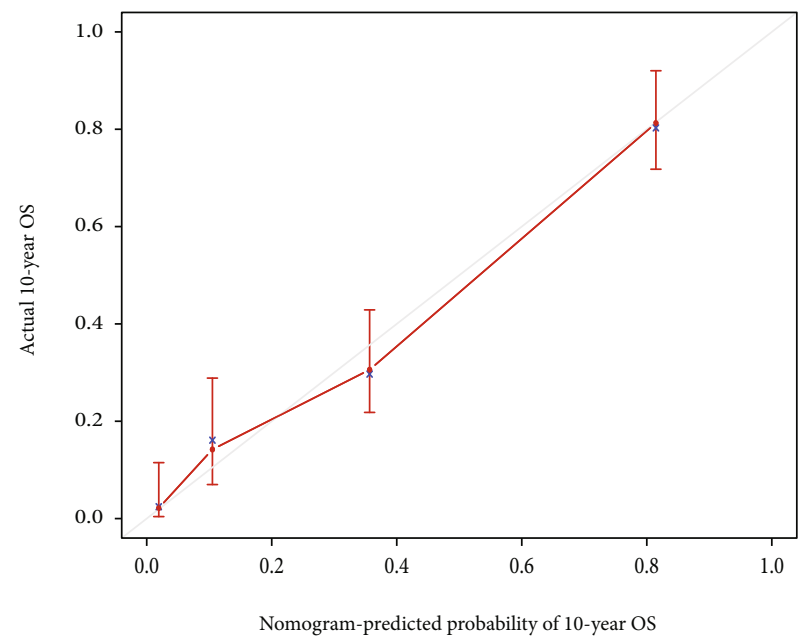

(c)

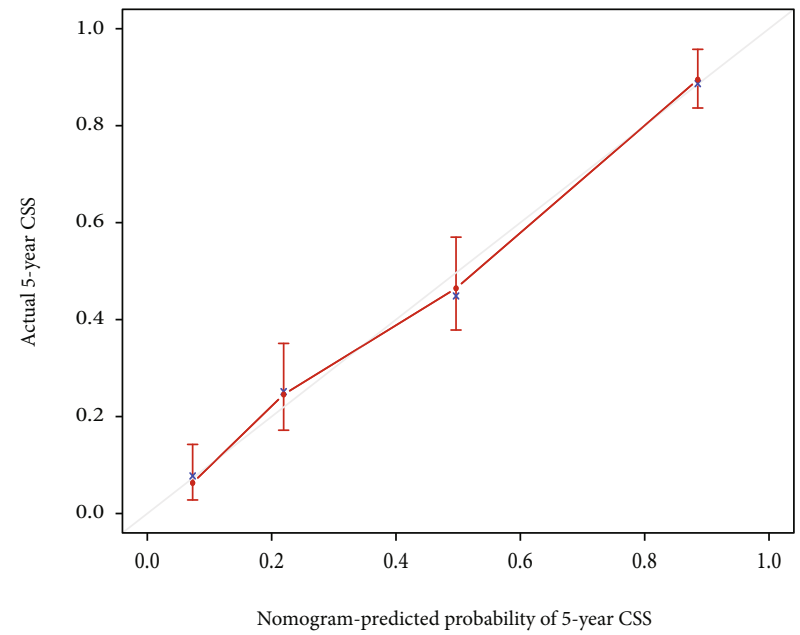

(e)

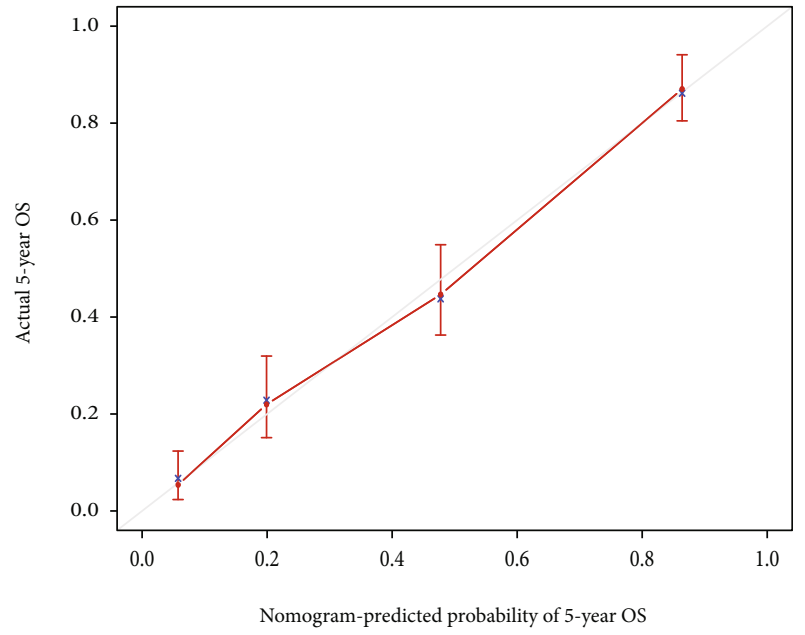

(b)

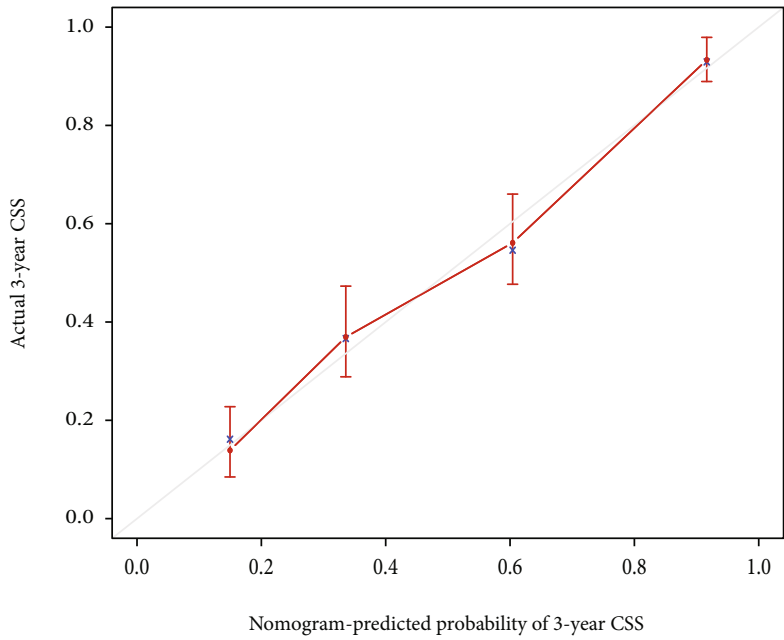

(d)

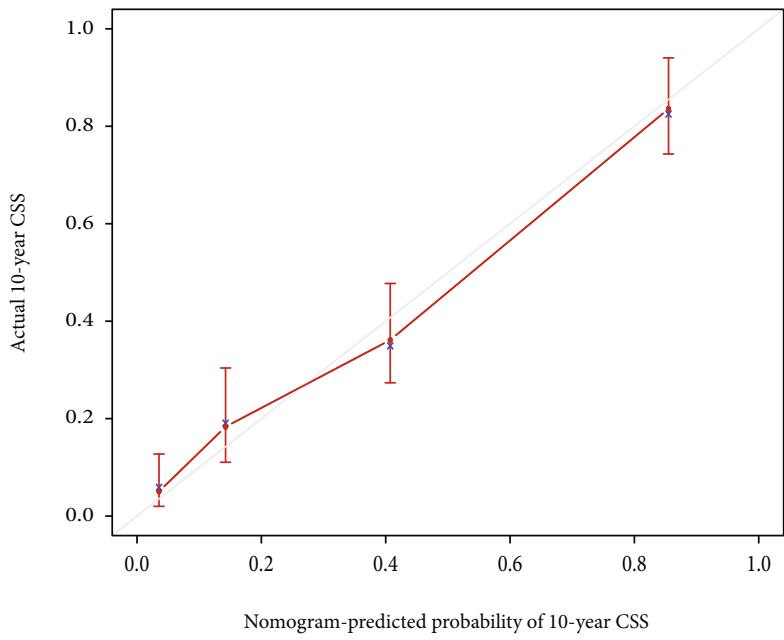

(f)

FIgURE 7: Calibration curves of the nomogram for 3-, 5-, and 10-year OS (a-c) and CSS (d-f) in testing cohort.

the prognosis had improved in Asian patients. All of these patients received surgery in this county, basically except for the survival effects of radical gastrectomy and expanded lymphadenectomy, which were more common in Asia [20].
Beyond all doubt, TNM stage and PLNE are internationally recognized prognostic factor.

Tumor size reflects tumor burden of tumor patients and is related to the burden and prognosis. A study of 946 


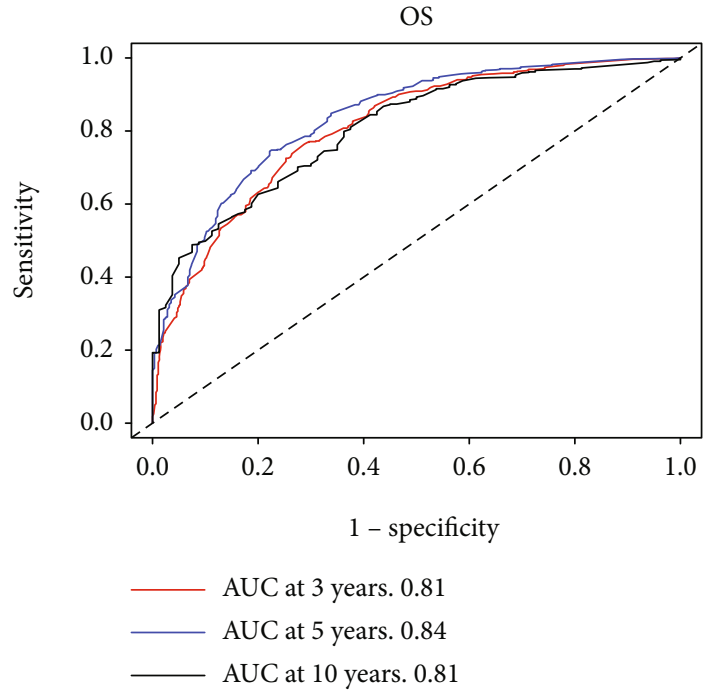

(a)

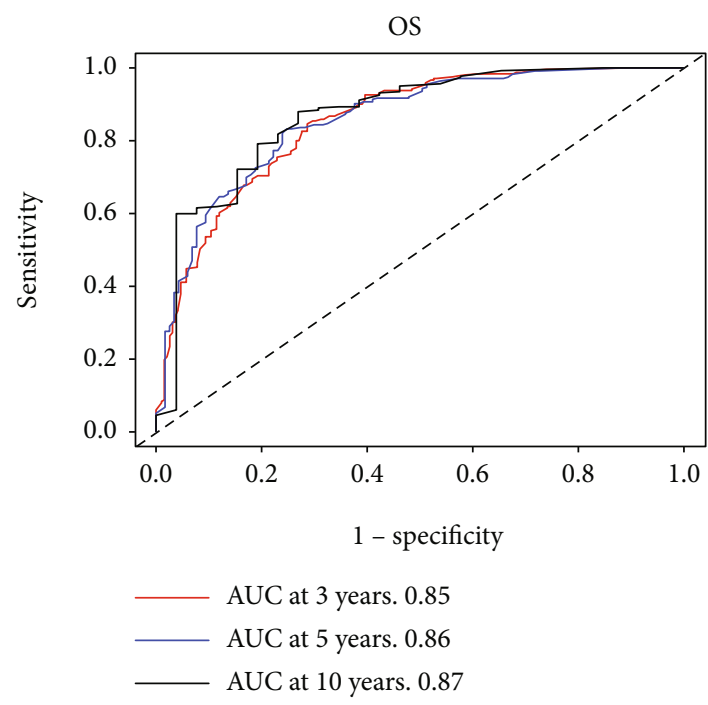

(c)

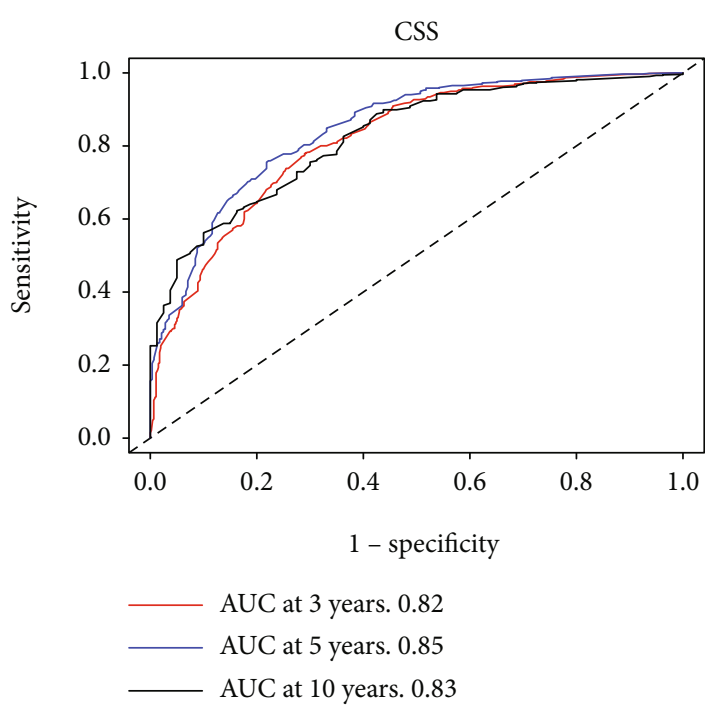

(b)

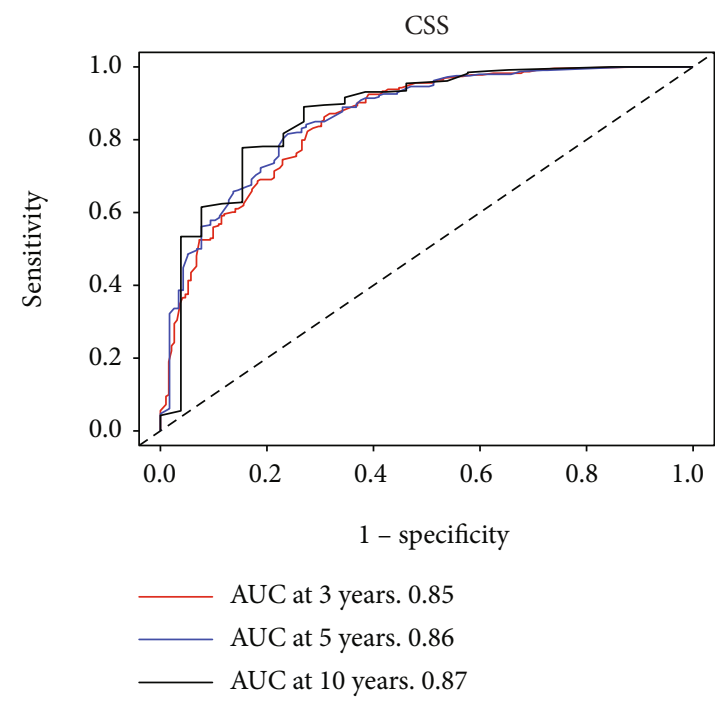

(d)

Figure 8: ROC curves for 3-, 5-, and 10-year OS and CSS in training $(a, b)$ and testing $(c, d)$ cohort.

patients with GSRC showed that the OS of small tumors (diameter less than $49 \mathrm{~mm}$ ) was better than that of large tumors (diameter greater than $49 \mathrm{~mm}$ ), and tumor size was an independent prognostic factor. In addition, lymph node metastasis, distant metastasis, and poor differentiation are the most likely to occur in the larger volume of GSRC, and these factors will affect the survival of patients $[8,21,22]$.

In recent years, the psychological intervention has gradually received attention in cancer treatment. Similar to our research results, many literatures pointed out that marital status was a socioeconomic factor that affects prognosis of cancer patients [23-26]. This may be married people get more humanistic care and emotional support to achieve better survival outcomes [27]. Although it was not an independent risk factor, it should be taken seriously.

Needless to state, surgery is the primary treatment for patients with GA, but many studies had proposed that chemotherapy can improve the prognosis of patients. Recent data indicated that paclitaxel-based chemotherapy had a definite effect in gastric signet ring cell carcinoma [28-30]. The advanced stage GSRC receiving TEFOX (docetaxel-5FU-oxaliplatin) chemotherapy had an effective rate of $65 \%$ and a median OS of up to 14 months [31]. We discovered that chemotherapy was an adverse prognostic factor by univariate COX regression; however, in multivariate COX regression, it was protective. Therefore, we analyzed the variables affecting chemotherapy and then found some significant differences between chemotherapy group and nonchemotherapy group, which were primary site, $\mathrm{T}$ stage, $\mathrm{N}$ stage, $\mathrm{RT}$, and tumor size. Further stepwise Cox regression showed that the $\mathrm{N}$ stage notably affected chemotherapy. That was to say, among the single factors, the worse prognosis of chemotherapy patients may be due to their later $\mathrm{N}$ stage. Consistent with our study, some articles also put forward that $\mathrm{N}$ stage was more senior in chemotherapy patients [32-35]. Despite the fact that GSRC is relatively insensitive to non-GSRC 
chemotherapy, it may benefit from specific chemotherapy regimens.

But for all this, our research still has several limitations that need to be pondered. First of all, in the SEER database, some important biochemical indexes were not explained, such as carcinoembryonic antigen (CEA) and carbohydrate antigen 19-9 (CA19-9). Differences in these factors may lead to differences in survival. Secondly, the information related to chemotherapy was not fully recorded, including chemotherapy regimen used, drugs dose, treatment duration, and adverse reaction. So it is not possible to find the best personal treatment. Additionally, we excluded some patients who were short of some information, which may lead to a deviation in prognosis analysis. Anyway, our nomograms were reliable individualized prediction models.

\section{Conclusion}

In conclusion, we firstly established and validated the nomograms for nonelderly adults with primary GSRC, which were used to forecast OS and CSS. Furthermore, the nomograms revealed excellent performance and strong predictive ability. This will provide theoretical support for the formulation of clinical treatment programs and prognostic judgment.

\section{Data Availability}

Data extracted from the SEER database do not require individual informed consent. The patient data in this study was anonymously managed in all stages, including stages of data cleaning and statistical analyses. This study was conducted in accordance with the Declaration of Helsinki.

\section{Conflicts of Interest}

The authors declare that there is no conflict of interest regarding the publication of this paper.

\section{Authors' Contributions}

(I) Hui Wang and Yao Peng contributed to the conception and design. (II) Mingjun Zhang contributed to the administrative support. (III) Hui Wang and Qi Huang contributed to the provision of study materials or patients. (IV) Hui Wang, Yao Peng, and Jinging Wu contributed to the collection and assembly of data. (V) Hui Wang, Yao Peng, Qi Huang, and Jingjing $\mathrm{Wu}$ contributed to the data analysis and interpretation. (VI) All authors contributed to the manuscript writing. (VII) All authors contributed to the final approval of manuscript. Hui Wang and Yao Peng contributed equally to this work as co-first authors

\section{Acknowledgments}

The authors would like to thank SEER for open access to the database. The present study was supported by the Anhui Natural Science Foundation of China (No. 1908085MH262, 2008085QH379) and the Medical Physics and Technology Key Laboratory of Anhui Province Open Fund (No. LMPT201907).

\section{Supplementary Materials}

Supplementary Table 1 shows the demographics and clinical characteristics of 1686 gastric signet ring cell carcinoma (GSRC) and 3060 non-GSRC. (Supplementary Materials)

\section{References}

[1] F. Bray, J. Ferlay, I. Soerjomataram, R. L. Siegel, L. A. Torre, and A. Jemal, "Global cancer statistics 2018: GLOBOCAN estimates of incidence and mortality worldwide for 36 cancers in 185 countries," CA: a Cancer Journal for Clinicians, vol. 68, no. 6, pp. 394-424, 2018.

[2] P. G. Chu and L. M. Weiss, "Immunohistochemical characterization of signet-ring cell carcinomas of the stomach, breast, and colon," American Journal of Clinical Pathology, vol. 121, no. 6, pp. 884-892, 2004.

[3] D. E. Henson, C. Dittus, M. Younes, H. Nguyen, and J. Albores-Saavedra, "Differential trends in the intestinal and diffuse types of gastric carcinoma in the United States, 19732000: increase in the signet ring cell type," Archives of Pathology \& Laboratory Medicine, vol. 128, no. 7, pp. 765-770, 2004.

[4] T. Voron, M. Messager, A. Duhamel et al., "Is signet-ring cell carcinoma a specific entity among gastric cancers?," Gastric Cancer, vol. 19, no. 4, pp. 1027-1040, 2016.

[5] L. M. Postlewait, M. H. Squires 3rd, D. A. Kooby et al., "The prognostic value of signet-ring cell histology in resected gastric adenocarcinoma," Annals of Surgical Oncology, vol. 22, no. S3, pp. 832-839, 2015.

[6] Z. Wang, J. Xu, Z. Shi et al., "Clinicopathologic characteristics and prognostic of gastric cancer in young patients," Scandinavian Journal of Gastroenterology, vol. 51, no. 9, pp. 1043-1049, 2016.

[7] F. J. Hsieh, Y. C. Wang, J. T. Hsu et al., "Clinicopathological features and prognostic factors of gastric cancer patients aged 40 years or younger," Journal of Surgical Oncology, vol. 105, no. 3, pp. 304-309, 2012.

[8] H. J. Park, J. Y. Ahn, H. Y. Jung et al., "Clinical characteristics and outcomes for gastric cancer patients aged 18-30 years," Gastric Cancer, vol. 17, no. 4, pp. 649-660, 2014.

[9] S. D. Nelen, R. H. A. Verhoeven, V. Lemmens, J. H. W. de Wilt, and K. Bosscha, "Increasing survival gap between young and elderly gastric cancer patients," Gastric Cancer, vol. 20, no. 6, pp. 919-928, 2017.

[10] L. H. Sobin, "TNM: evolution and relation to other prognostic factors," Seminars in Surgical Oncology, vol. 21, no. 1, pp. 3-7, 2003.

[11] Y. Wang, Z. Pang, X. Chen, T. Yan, J. Liu, and J. Du, "Development and validation of a prognostic model of resectable smallcell lung cancer: a large population-based cohort study and external validation," Journal of Translational Medicine, vol. 18, no. 1, p. 237, 2020.

[12] C. Fang, W. Wang, X. Feng et al., "Nomogram individually predicts the overall survival of patients with gastroenteropancreatic neuroendocrine neoplasms," British Journal of Cancer, vol. 117, no. 10, pp. 1544-1550, 2017.

[13] L. Huang, Y. Balavarca, L. van der Geest et al., "Development and validation of a prognostic model to predict the prognosis of patients who underwent chemotherapy and resection of pancreatic adenocarcinoma: a large international populationbased cohort study," BMC Medicine, vol. 17, no. 1, p. 66, 2019. 
[14] K. A. Cronin, L. A. Ries, and B. K. Edwards, "The Surveillance, Epidemiology, and End Results (SEER) Program of the National Cancer Institute," Cancer, vol. 120, Suppl 23, pp. 3755-3757, 2014.

[15] F. D. Huitzil-Melendez, M. Capanu, E. M. O'Reilly et al., "Advanced hepatocellular carcinoma: which staging systems best predict prognosis?," Journal of Clinical Oncology, vol. 28, no. 17, pp. 2889-2895, 2010.

[16] Z. Lei, J. Li, D. Wu et al., "Nomogram for preoperative estimation of microvascular invasion risk in hepatitis B virus-related hepatocellular carcinoma within the Milan criteria," JAMA Surgery, vol. 151, no. 4, pp. 356-363, 2016.

[17] X. R. Tang, Y. Q. Li, S. B. Liang et al., "Development and validation of a gene expression-based signature to predict distant metastasis in locoregionally advanced nasopharyngeal carcinoma: a retrospective, multicentre, cohort study," The Lancet Oncology, vol. 19, no. 3, pp. 382-393, 2018.

[18] X. F. Yang, L. Yang, X. Y. Mao, D. Y. Wu, S. M. Zhang, and Y. Xin, "Pathobiological behavior and molecular mechanism of signet ring cell carcinoma and mucinous adenocarcinoma of the stomach: a comparative study," World Journal of Gastroenterology, vol. 10, no. 5, pp. 750-754, 2004.

[19] A. Wang, M. H. Squires 3rd, M. Melis et al., "Stage-specific prognostic effect of race in patients with resectable gastric adenocarcinoma: an 8-institution study of the US gastric cancer collaborative," Journal of the American College of Surgeons, vol. 222, no. 4, pp. 633-643, 2016.

[20] J. Kim, C. L. Sun, B. Mailey et al., "Race and ethnicity correlate with survival in patients with gastric adenocarcinoma," Annals of Oncology, vol. 21, no. 1, pp. 152-160, 2010.

[21] L. Zhou, W. Li, S. Cai, C. Yang, Y. Liu, and Z. Lin, "Large tumor size is a poor prognostic factor of gastric cancer with signet ring cell: results from the surveillance, epidemiology, and end results database," Medicine (Baltimore), vol. 98, no. 40, article e17367, 2019.

[22] J. H. Pyo, H. Lee, Y. W. Min et al., "Indication for endoscopic treatment based on the risk of lymph node metastasis in patients with Siewert type II/III early gastric cancer," Gastric Cancer, vol. 21, no. 4, pp. 672-679, 2018.

[23] M. Reyngold, K. A. Winter, W. F. Regine et al., "Marital status and overall survival in patients with resectable pancreatic cancer: results of an ancillary analysis of NRG oncology/RTOG 9704," The Oncologist, vol. 25, no. 3, pp. e477-e483, 2020.

[24] G. Inverso, B. A. Mahal, A. A. Aizer, R. B. Donoff, N. G. Chau, and R. I. Haddad, "Marital status and head and neck cancer outcomes," Cancer, vol. 121, no. 8, pp. 1273-1278, 2015.

[25] S. L. Gomez, S. Hurley, A. J. Canchola et al., "Effects of marital status and economic resources on survival after cancer: a population-based study," Cancer, vol. 122, no. 10, pp. 1618$1625,2016$.

[26] J. J. Jin, W. Wang, F. X. Dai et al., "Marital status and survival in patients with gastric cancer," Cancer Medicine, vol. 5, no. 8, pp. 1821-1829, 2016.

[27] M. S. Rendall, M. M. Weden, M. M. Favreault, and H. Waldron, "The protective effect of marriage for survival: a review and update," Demography, vol. 48, no. 2, pp. 481-506, 2011.

[28] S. Kim, F. Fiteni, S. Paget-Bailly et al., "The impact of taxanebased preoperative chemotherapy in gastroesophageal signet ring cell adenocarcinomas," Journal of Hematology \& Oncology, vol. 8, no. 1, p. 52, 2015.
[29] L. Chen, Y. Shi, J. Yuan et al., "Evaluation of docetaxel- and oxaliplatin-based adjuvant chemotherapy in postgastrectomy gastric cancer patients reveals obvious survival benefits in docetaxel-treated mixed signet ring cell carcinoma patients," Medical Oncology, vol. 31, no. 9, p. 159, 2014.

[30] S. E. al-Batran, R. D. Hofheinz, C. Pauligk et al., "Histopathological regression after neoadjuvant docetaxel, oxaliplatin, fluorouracil, and leucovorin versus epirubicin, cisplatin, and fluorouracil or capecitabine in patients with resectable gastric or gastro-oesophageal junction adenocarcinoma (FLOT4AIO): results from the phase 2 part of a multicentre, openlabel, randomised phase 2/3 trial," The Lancet Oncology, vol. 17, no. 12, pp. 1697-1708, 2016.

[31] S. Pernot, O. Dubreuil, T. Aparicio et al., "Efficacy of a docetaxel-5FU-oxaliplatin regimen (TEFOX) in first-line treatment of advanced gastric signet ring cell carcinoma: an AGEO multicentre study," British Journal of Cancer, vol. 119, no. 4, pp. 424-428, 2018.

[32] P. Gabani, J. Barnes, A. J. Lin et al., "Induction chemotherapy in the treatment of nasopharyngeal carcinoma: clinical outcomes and patterns of care," Cancer Medicine, vol. 7, no. 8, pp. 3592-3603, 2018.

[33] S. Nazzani, F. Preisser, E. Mazzone et al., "Survival effect of perioperative systemic chemotherapy on overall mortality in locally advanced and/or positive regional lymph node nonmetastatic urothelial carcinoma of the upper urinary tract," World Journal of Urology, vol. 37, no. 7, pp. 1329-1337, 2019.

[34] X. B. Pan, L. Li, S. Qu, L. Chen, S. X. Liang, and X. D. Zhu, “The efficacy of chemotherapy in survival of stage II nasopharyngeal carcinoma," Oral Oncology, vol. 101, p. 104520, 2020.

[35] X. Wang, C. Yin, S. Su et al., "Long-term effects of neoadjuvant radiotherapy, adjuvant radiotherapy, and chemotherapy-only on survival of locally advanced non-small cell lung cancer undergoing surgery: a propensity-matched analysis," BMC Cancer, vol. 18, no. 1, p. 1067, 2018. 